\title{
Modelling the interfacial behaviour of dilute light-switching surfactant solutions
}

\author{
Carmelo Herdes, ${ }^{\text {a }}$ Erik E. Santiso, ${ }^{\mathrm{b}}$ Craig James, ${ }^{\mathrm{c}}$ Julian Eastoe ${ }^{\mathrm{d}}$ and Erich A. \\ Müller ${ }^{\mathrm{a}, ~ *}$ \\ ${ }^{a}$ Department of Chemical Engineering, Imperial College London, London, SW7 2AZ, U.K. \\ ${ }^{b}$ Department of Chemical and Biomolecular Engineering, North Carolina State University, \\ NC 27695-7905, U.S.A.
}

${ }^{c}$ Department of Frontier Materials Chemistry, Graduate School of Science and Technology, Hirosaki University, 3 Bunkyo-cho, Hirosaki, Aomori 036-8561, Japan

${ }^{d}$ School of Chemistry, University of Bristol, Bristol BS8 1TS, U.K.

* corresponding author: e.muller@imperial.ac.uk

\begin{abstract}
The direct molecular modelling of an aqueous surfactant system at concentrations below the critical micelle concentration (pre-cmc) conditions is unviable in terms of the presently available computational power. Here, we present an alternative that combines experimental information with tractable simulations to interrogate the surface tension changes with composition and the structural behaviour of surfactants at the water-air interface. The methodology is based on the expression of the surface tension as a function of the surfactant surface excess, both in the experiments and in the simulations, allowing direct comparisons to be made. As a proof-of-concept a coarse-grained model of a light switching non-ionic surfactant bearing a photosensitive azobenzene group is considered at the air-
\end{abstract}


water interface at $298 \mathrm{~K}$. Coarse-grained molecular dynamic simulations are detailed based on the use of the SAFT- $\gamma$-Mie Force Field with parameters tuned specifically for this purpose. An excellent agreement is obtained between the simulation predictions and experimental observations; furthermore, the molecular model allows the rationalization of the macroscopic behaviour in terms of the different conformations of the cis and trans surfactants at the surface.

Keywords: Molecular dynamics, surface tension, molecular simulation, coarse graining, SAFT, amphiphiles, complex fluids, CMC.

\section{Introduction}

Surface tension is a central property of interest in solutions of surfactants and amphiphiles, and knowledge of its behaviour as a function of temperature, pressure and concentration is a key factor to evaluate the performance of consumer household products, biocompatible drug delivery systems, additives for enhanced gas solubility and oil recovery, just to name a few. ${ }^{1,2}$

The capacity of surfactants for lowering the surface tensions of aqueous solutions can be discussed in terms of (i) the concentration required to produce a given surface tension reduction and (ii) the maximum reduction in surface tension that can be obtained regardless of concentration. ${ }^{3}$ These are referred to as the surfactant efficiency and effectiveness respectively. As a rule of thumb, a good measure of the surfactant adsorption efficiency is the concentration of surfactant required to produce a $20 \mathrm{mN} \mathrm{m}^{-1}$ reduction in surface tension. At this value, typically the surfactant concentration is close to the minimum concentration needed to produce maximum adsorption at the interface. The performance of a given surfactant can also be discussed in terms of effectiveness of 
adsorption at the air-water interface. The effectiveness of adsorption is an important factor in determining properties such as foaming, wetting, and emulsification. This is usually quantified as the maximum lowering of surface tension $\gamma_{\min }$ (regardless of concentration), or as the surface excess concentration at surface saturation equivalent to the maximum adsorption, $\Gamma_{\max }$, (a measure of the interfacial packing). For non-ionic surfactants, $\gamma_{\min }$, and $\Gamma_{\max }$, happen to closely match the critical micelle concentration $(\mathrm{cmc})$, above which surfactants self-assemble in the bulk water phase. To complicate matters further, the efficiency and effectiveness of surfactants do not necessarily run parallel, and it is commonly observed - as shown by Rosen's extensive data listing ${ }^{4}-$ that materials producing significant lowering of the surface tension at low concentrations (i.e., they are more efficient) have smaller $\Gamma_{\max }$ (i.e., they are less effective).

At the molecular level, surfactant efficiency is mainly dictated by energetics, whereas, its role in effectiveness is directly related to entropic effects, i.e. to the relative size of the hydrophilic and hydrophobic portions of the adsorbing molecules. The area occupied by each molecule is determined either by the hydrophobic chain cross-sectional area, or the area required for closest packing of head groups, whichever is greater. Therefore, surfactant films can be tightly or loosely packed resulting in very different interfacial properties. For instance, straight chains and large head groups (relative to the tail cross section) favour close, effective packing, whereas branched, bulky, or multiple hydrophobic chains give rise to steric hindrance at the interface. This competition between energetic and entropic contributions is the main driving force for the interfacial behaviour of surfactants and may lead to the observation of surface phase transitions at the interfaces, ${ }^{5}$ including liquid crystal-like dense 2D phase upon compression. 
It is a non-trivial task to deduce the mesophase behaviour of dilute surfactants solutions from the above-mentioned heuristic arguments, and no accepted predictive tool exists for this purpose, hence the increased relevance on experimental probing of surfactant systems. Simple experimental approaches can be implemented for the measurement of the air-water surface tensions in dilute surfactant solutions; however, they only provide indirect evidence of the surface filling by surfactants. To probe the surfactant layer structures and self-assembly at the water-air interfaces direct scattering methods (e.g. neutron reflection) are routinely used, ${ }^{6}$ however, they are not without complications and limitations.

It would seem sensible to interrogate these systems employing molecular simulation. For recent reviews of the current perspective on the applications of molecular modeling in the present context the reader is referred to the reviews by Maginn and Elliott ${ }^{7}$ and in particular to the recent one by Creton et al., ${ }^{8}$ focused on surfactant solutions. In spite of the obvious insights that molecular modeling can deliver, the straightforward atomistic modelling of a surfactant system at pre-cmc conditions is unviable in terms of the present (and for the foreseeable future) computational capacity. As an example, consider a typical non-ionic surfactant, which ca. 60 atoms immersed in a water solution. The concentration range in the pre-cmc region spans from infinite dilution up to $O\left(10^{-1} \mathrm{~mol} \mathrm{~m}^{-3}\right)$. The smallest simulation cell to mimic the latter state point would require $O\left(10^{5}\right)$ water molecules per surfactant. If one wishes to model the saturated surface along with surfactants in the bulk and/or several micelles then the system size runs into the $O\left(10^{7}\right)$ atom sites. Furthermore, to guarantee an equilibrated state and suitable statistics, simulations need to be run for at least several hundreds of nanoseconds to explore the diffusion dynamics. This is not within the realm of the simulations possible with conventional hardware in terms of both system size and simulation length. The most 
discouraging point is that in this scenario, due to the dilution of the system, the vast majority of the computations are spent in modelling the bulk behaviour of pure water, which, in this context, is superfluous.

It is not to say that "heroic" atomistic simulations have not been attempted to study a variety of surfactant micellar systems ${ }^{9-21}$ and insights have been gained regarding structural properties of post-cmc regions through calculation of density distributions, micelle size and shapes, average micellar population, etc. The challenge remains in tackling the computationally demanding calculations in the pre-cme region. Coarse-grained (CG) methodologies have been used to approach these systems, reducing the number of nonrelevant degrees of freedom and allowing for more tractable simulations, ${ }^{22-29}$. However, even with commonly used CG approaches, pre-cmc calculations are strongly dependent on (i) the capability of the model to preserve the molecular character of surfactant-solvent interactions and, (ii) the adopted methodology. ${ }^{30-38}$ However, in these simulations, the key limitation to performing a full-fledged simulation including the surfactant and the full amount of solvent molecules associated with these dilute conditions remains, even with the reduced resolution of the coarse grained models.

An underlying aim of this communication is to present an alternative simulation model to explore the pre-cmc region in a surfactant at the free air-water interface. Having the typical experimental setup in mind we propose the use of a representative simulation cell to model this system in a tractable time span based on the calculation of the surface tension in terms of the surface excess data. The key point is to recognize that in the dilute regime, the concentration of most surfactants, and certainly the non-ionic ones in the bulk solution is negligible as compared to the accumulation at the free surface, hence the 
accounting of the bulk phase adds no new information. The methodology is showcased by exploring the interfacial behaviour of light-switching surfactants.

\section{Surface tension isotherms}

Experimentally, the outcome of tensiometry is the generation of a surface tension isotherm, providing the variation of surface tension with concentration. The plot will typically have a shape similar to that seen in the left hand side of figure 1 . The tension decreases with concentration until a limiting value is obtained, where the increase in surfactant concentration does not alter the tension (to a first approximation). The challenge from a molecular simulation perspective is to model this curve, as the concentrations involved are in a very dilute regime.

The description of this system in terms of classical thermodynamics is very well known and the reader is referred to the classical textbooks on the subject, among then are, ref. 3, 4 and 39. Only a few relevant concepts will be briefly described herein to explain the methodology. The starting point is the Gibbs adsorption isotherm expressing the relationship that must hold at a given constant temperature and pressure between the surface tension, the chemical potential of one of the $i$ species, $\mu_{i}$, and number of moles, $n_{i}^{s}$, of each of the $k$ components within an arbitrary region of area $A$ defined as an interface, $s$, that separates two bulk regions $\alpha$ and $\beta$,

$$
\sum_{i=1}^{k} n_{i}^{s} d \mu_{i}+A d \gamma=0
$$

It is convenient to express the compositions in terms of an intensive quantity, the surface excess, $\Gamma$, defined as 


$$
\Gamma_{i}=\frac{n_{i}^{s}}{A}=\frac{n_{i}^{\text {total }}-n_{i}^{\alpha}-n_{i}^{\beta}}{A}
$$

For a binary system consisting of a solvent (1), and a solute (2), i.e. the subscript 1 refers to water and 2 to the surfactant; equation (1) then reduces to

$$
-d \gamma=\Gamma_{1} d \mu_{1}+\Gamma_{2} d \mu_{2}
$$

The choice of the exact location of the interface is to some extent arbitrary. Considering the Gibbsian election of dividing surface position, one such there is no net adsorption of solvent, $\Gamma_{1}=0$, then equation (3) simplifies to

$$
d \gamma=-\Gamma_{2} d \mu_{2}
$$

For simplicity, indices can be dropped and the surfactant surface concentration at the airwater interface can be identified by $\Gamma$.

It can be safely assumed that the surfactant will be diluted to the extent that it complies with ideal solution behaviour and $d \mu=R T d \ln (c)$ were $R$ is the gas constant, $T$ is the system temperature and $c$ is the surfactant concentration. Then, for a charge neutral surfactant, (4) can be rewritten as:

$$
\Gamma=-\frac{1}{R T} \frac{d \gamma}{d \ln (c)}
$$

Equation (5) relates the surface excess to the derivative of the surface tension isotherm. Hence, one could use the isothermal data in the pre-cmc region where tangents of the plot correspond to the local tangent $\left(\frac{d \gamma}{d \ln (c)}\right)$, to produce a curve that relates the surface 
excess to the bulk surfactant concentration (c.f. figure 1 top right). Experimentally, this procedure has been proved to work well for $\mathrm{C}_{\mathrm{i}} \mathrm{E}_{\mathrm{j}} \mathrm{OH}$ nonionic surfactants, finding good agreements between $\Gamma$ values from tensiometry and using equation (5) with those measured directly by neutron reflection. ${ }^{5}$

For this purpose however, a more interesting plot is that of the surface tension in terms of the surface excess (figure 1, bottom right), which again is acquired from the same data. This plot, derived from the adsorption isotherm, does not have in an explicit fashion the information of the bulk phase concentration and relates the surface tension directly to the properties of the surfactant at the water-air interface. From the simulation point of view, this is a crucial abstraction, as one can probe a nanoscopic section of this interface with the appropriate detail, calculating independently both the surface tension and the surface concentration. The novelty of the methodology used herein is in the actual abstraction based on exploring $\Gamma$ rather than surfactant concentration. 


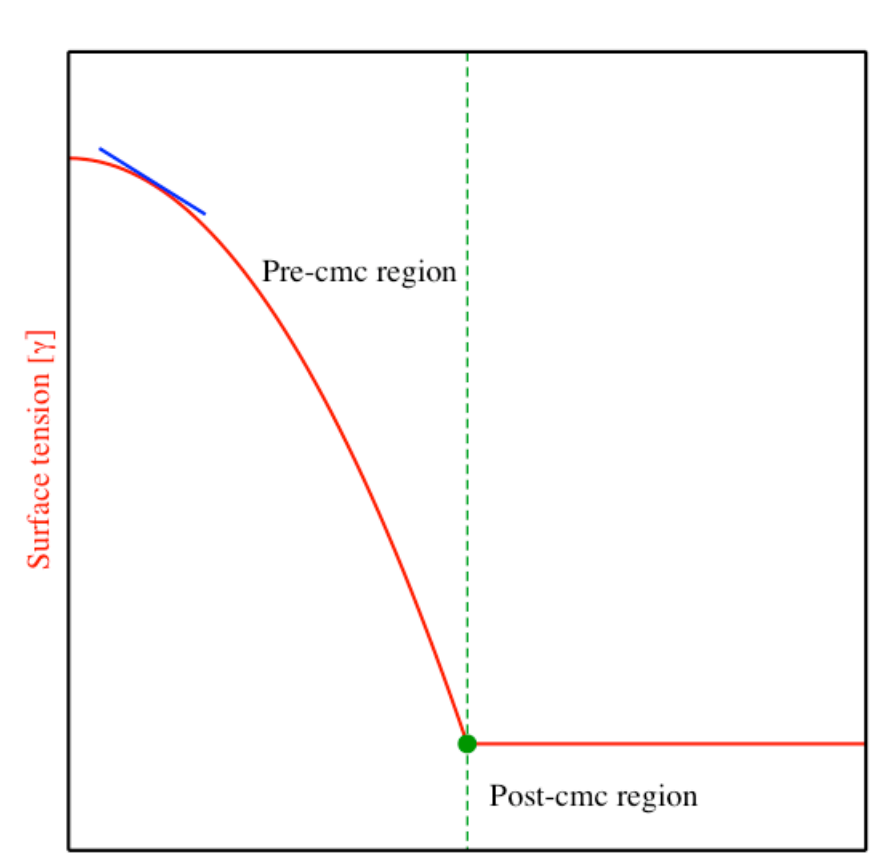

Surfactant concentration [c]

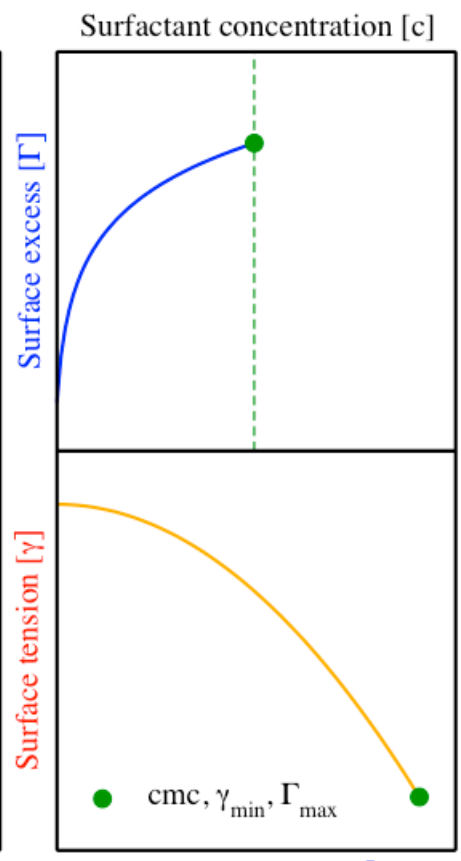

Surface excess $[\Gamma]$

Figure 1. Schematic representation of surface tension concentration plot and its transformation into a $\gamma-\Gamma$ plot. The slope of the traditional surface tension isotherm (blue line in the left diagram) corresponds to the surface excess, which can then be represented either as a function of the surfactant concentration (upper right diagram), or more interestingly, on a concentration-free basis by plotting it in terms of the tension (lower right diagram).

\section{Molecular model of photo-sensitive surfactants.}

The concept of photo-driven surfactancy was developed by Shinkai ${ }^{40}$, apparently the first to suggest that the incorporation of an azobenzene group into the hydrophobic chains of a surfactant could affect the hydrophilic-lipophilic balance if perturbed through light-driven isomerization. The general concept is most interesting, as it suggests that one would be able to drive reversible adsorption-desorption phenomena, and stability-instability transitions in emulsions, for example. A recent review $^{41}$ presents some of the recent developments and challenges in this field. Some of the most detailed experimental work to date on these systems can be traced to the Hatton group ${ }^{42}$ which studied non-ionic photosensitive surfactants consisting of a polar di(ethylene oxide) head group attached to an 
alkyl spacer of between two and eight methylene groups, coupled through an ether linkage to an azobenzene moiety. An example of such molecule is seen in figure 2. There is experimental evidence of the structural changes associated with the interconversion of the azobenzene group between its cis and trans forms, mediated by the appropriate wavelength of an irradiating light source. This interconversion causes changes in the surface tension and critical micellization concentrations ${ }^{42}$.

As a proof of concept of the proposed methodology, a light switching surfactant of the above mentioned family, diethylene glycol mono (4',4-hexyloxy, butyl-azobenzene), $\mathrm{C}_{4} \mathrm{AzzOCC}_{6} \mathrm{E}_{2}$, is considered at the air-water interface at $298 \mathrm{~K}$. The predominantly cis isomer exhibits a surface tension of $40.6 \mathrm{mN} \mathrm{m}^{-1}$ at the $\mathrm{cmc}$ at $4.5 \times 10^{-3} \mathrm{~mol} \mathrm{~m}^{-3}$, meanwhile, the predominantly trans structure shows a lower surface tension, $29.5 \mathrm{mN} \mathrm{m}^{-1}$ at a lower cmc of $1.0 \times 10^{-3} \mathrm{~mol} \mathrm{~m}^{-3}{ }^{42}$

We chose to coarse-grain the surfactant in order to access both the time and length scales required for the modelling of the system. For this purpose, the SAFT- $\gamma$ force field ${ }^{43}$ is employed. This coarse-grained methodology maps the average energetic and structural properties of small chemical moieties to a simplified bead-chain model through the use of a molecular based equation of state. In essence, each bead is a coarse grained representation of a group of atoms. Surfactant molecules may be recast at this level by employing a chain of tangent-beads: a triplet bead labelled $\mathrm{C}$, which groups the terminal methyl carbitol $\left[\mathrm{CH}_{3}-\mathrm{CH}_{2}-\mathrm{O}-\mathrm{CH}_{2}-\mathrm{CH}_{2}-\mathrm{O}-\mathrm{CH}_{2}-\mathrm{CH}_{2}-\mathrm{OH}\right]$, a bead labelled P, fitted to propane - $\left[\mathrm{CH}_{2}-\mathrm{CH}_{2}-\right.$ $\left.\mathrm{CH}_{2}\right]$ - or the terminal $-\left[\mathrm{CH}_{2}-\mathrm{CH}_{2}-\mathrm{CH}_{3}\right]$ groups, a triplet bead labelled $\mathrm{A}$, which groups anisol $-\left[\mathrm{CH}_{2}-\mathrm{O}-\mathrm{C}_{6} \mathrm{H}_{4}\right]-$, a double $\mathrm{T}$ bead fitted to toluene $-\left[\mathrm{CH}_{2}-\mathrm{C}_{6} \mathrm{H}_{4}\right]-$ and a single bead $\mathrm{N}$ for the nitrogen molecule. Figure 2 shows a cartoon of the CG model superimposed to the 
atomistic depiction for reference purposes only. It is obvious that the breakdown of the surfactant into the groups chosen is completely arbitrary and other choices could have been made. No attempt was made to optimize these choices, and in general the objective of said breakdown is to be able to assign to each CG bead intermolecular parameters, which are in the best possible measure quantitative representations of the average energetic and structural properties. Another detail is that the breakdown of the molecules in terms of smaller groups assigned to common small molecules neglects in cases of the connectivity a $\mathrm{H}$ atom which is replaced by a bond, e.g. the group $\mathrm{C}_{6} \mathrm{H}_{4}-\mathrm{CH}_{2}$ is fitted to toluene, $\mathrm{C}_{6} \mathrm{H}_{5^{-}}$ $\mathrm{CH}_{3}$. We assume that these missing $\mathrm{H}$ do not affect the overall energetics. Water molecules are fitted to a single isotropic bead.

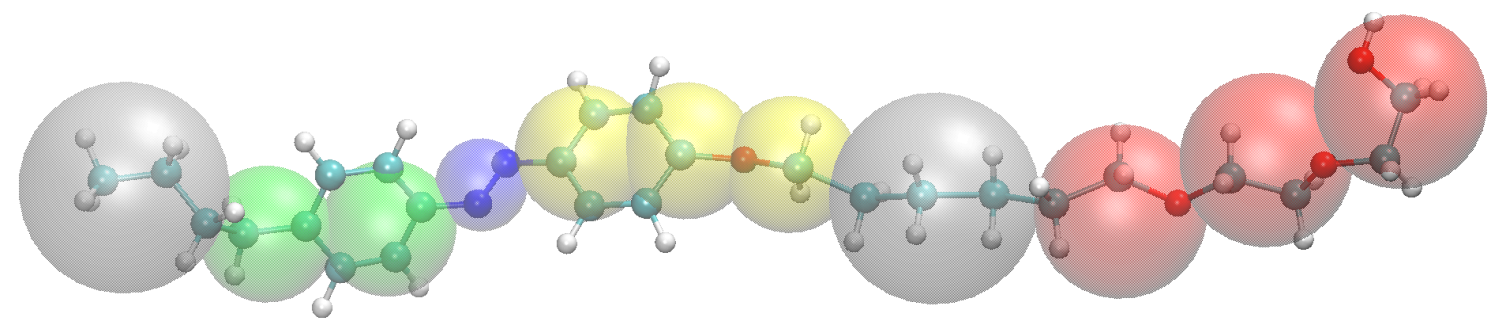

Figure 2. A cartoon of the CG model. The underlying atomistic depiction is placed as a reference, although the force field parameters are not obtained from the atomistic model, but from a top-down approach, from left to right, propane (grey), toluene (green), nitrogen (blue), anisole (yellow), propane, methyl carbitol (red).

The parameterization was carried out using a correlation based on the SAFT- $\gamma$ Mie $\operatorname{approach}^{44}$, where the non-bonded parameters for each group are obtained terms of their different functionalities within the surfactant molecule (see table 1). The interactions between unlike groups were obtained following the combining rules suggested by Lafitte et al. $^{44}$, with the exception of the well-depth energy parameter between the surfactant and water molecules, which was determined by comparing the model predictions to experimental data of the cis isomer. The SAFT- $\gamma$ forcefield is based on the mapping 
between a molecular equation of state and the effective forcefield from which it derives. Here the CG beads are represented by a Mie potential, $u$,

$$
u(r)=\left(\frac{\lambda_{r}}{\lambda_{r}-\lambda_{a}}\right)\left(\frac{\lambda_{r}}{\lambda_{a}}\right)^{\lambda_{a} /\left(\lambda_{r}-\lambda_{a}\right)} \varepsilon\left[\left(\frac{\sigma}{r}\right)^{\lambda_{r}}-\left(\frac{\sigma}{r}\right)^{\lambda_{a}}\right]=A r^{-\lambda_{r}}-\operatorname{Cr}^{-\lambda_{a}}
$$

where $r$ is the intermolecular distance, and $\varepsilon$, and $\sigma$, and are the adjustable parameters relating to the energy and distance scales. Noteworthy is that while the dispersion exponent was throughout fixed at the value of $\operatorname{six}\left(\lambda_{\mathrm{a}}=6\right)$, the short-range repulsion $\left(\lambda_{\mathrm{r}}\right)$ adopted different values reflecting the average softness/hardness (range) of the potential. More details about the CG procedure may be found elsewhere. ${ }^{45-47}$ The Mie potential in equation (6) may expressed in terms of two constants $A$ and $C$ that consolidate all the functionality corresponding to the prefactor, and the size and energy parameters. This functional form, expressed in the right hand side of equation (6) is commonly used when tabulating potentials in MD codes.

A final assumption regards to the connectivity between the beads, which in this case is a bond length equivalent to the van der Waals radii, $\sigma_{i j}$. One must bear in mind that the size parameters is not obtained by fitting to atomistic models, but rather using a top-down approach where the size of the beads are directly linked to the observed macroscopic density, hence a direct mapping to the detailed structure is not relevant. The intramolecular interactions are described by a harmonic potential that accounts for bond angle bending between three adjacent beads,

$$
U_{\text {intra }}=\sum^{\text {Angle }} k_{a}\left(\theta-\theta_{0}\right)^{2}
$$


where $k_{a}=2.1113\left[\mathrm{kcal} \mathrm{mol}^{-1} \mathrm{rad}^{-2}\right]$ is the bending spring constant and $\theta_{0}=157.6^{\circ}$ is the equilibrium angle, these values are used throughout unless otherwise noted. Here is were we choose to control the light switching surfactant behaviour, by fixing an angle of $45^{\circ}$ in the azo-benzene (benzene-nitrogen-benzene) group for the cis isomer and $180^{\circ}$ for the trans one, in all molecules.

Table 1. Non-bonded coarse-grained parameters

\begin{tabular}{|c|c|c|c|c|}
\hline Bead & $\sigma[\mathrm{nm}]$ & $\varepsilon / k_{B}[K]$ & $\lambda_{r}$ & $m^{(a)}$ \\
\hline Propane $(\mathrm{P})$ & 0.4871 & 426.08 & 34.29 & 1 \\
\hline Toluene $(\mathrm{T})$ & 0.4266 & 411.87 & 16.95 & 2 \\
\hline Nitrogen $(\mathrm{N})$ & 0.3653 & 122.85 & 20.02 & 1 \\
\hline Anisole (A) & 0.3751 & 350.30 & 14.31 & 3 \\
\hline Carbitol (C) & 0.4200 & 552.47 & 40.69 & 3 \\
\hline Water (W) & 0.2915 & 378.87 & 8.40 & 1 \\
\hline P-W & 0.3893 & 268.90 & 15.99 & \multirow{5}{*}{$\mathrm{n} / \mathrm{a}$} \\
\hline $\mathrm{T}-\mathrm{W}$ & 0.3591 & 276.20 & 11.68 & \\
\hline $\mathrm{N}-\mathrm{W}$ & 0.3284 & 156.21 & 12.58 & \\
\hline $\mathrm{A}-\mathrm{W}$ & 0.3333 & 262.54 & 10.81 & \\
\hline C-W & 0.3558 & 426.60 & 17.26 & \\
\hline
\end{tabular}

Notes

a) The parameter $m$, corresponding to the number of beads, taken as an integer, describes the molecule in terms of a chain of that length, i.e. $m=2$ is a dimer. Values correspond to each of the constituent beads.

b) The attractive exponent is kept at $\lambda_{a}=6$ for all cases.

c) Parameters are calculated using the procedures outlined in ref. 48.

\section{Molecular simulation details}

For the description of the pre-cmc region, a parallelepiped simulation box with aspect ratio $L_{z} / L_{x}=6$ was used, where $L_{x}=L_{y}=6 \mathrm{~nm}$. 16000 water molecules were employed; the number of surfactant molecules was varied from 1 to 150 per surface. This unit cell, initially filled with water molecules, is much larger than the one needed for a pure liquid phase; hence a slab of liquid with two interfaces is stabilized and coexists with a 
water vapour phase. At these conditions, the density of the water vapour is several orders of magnitude less than that of the liquid; hence the vapour phase is in essence a vacuum. Surfactant molecules were initially placed randomly in the void spaces of the cell, but rapidly migrated and collected at the surfaces of the aqueous slab. Larger systems with $L_{x}=L_{y}=12 \mathrm{~nm}$ and 64000 water molecules were tested with no appreciable difference in the results.

The system was run under a molecular dynamics canonical (NVT) ensemble, where the total volume, concentration and temperature are kept constant. The simulations were thermostated to $298 \mathrm{~K}$ every $1 \mathrm{ps}$ by a Nose-Hoover algorithm, all non-bonded interactions were truncated at $1.1 \mathrm{~nm}$. The GROMACS simulation open source suite ${ }^{49}$ was used to calculate the molecular dynamics. The systems were run with a time-step of $0.01 \mathrm{ps}$ for at least $10 \mathrm{~ns}$. It should be noted that due to the CG nature of the force fields, the dynamics of the system are also accelerated; hence 10 ns would correspond to a simulation of roughly $0.01 \mu \mathrm{s}$ if an all atom approach would have been used $^{50}$. All reported properties came from relevant averages, taken over the last half of the configurations explored.

In a canonical (constant number of particles, volume and temperature) ensemble, one may calculate the surface tension, $\gamma$, directly from a molecular dynamics simulation. There are essentially two routes to the determination of the surface tension; the most common one explores the relationship between elements of the pressure tensor, or mechanical route. A recent development consists of relating the tension to the results of a perturbation approach, sometimes referred to as the Test Area Method or thermodynamic route $^{51}$. In the limit of a planar interface both methods yield identical results. ${ }^{52}$ In most "off the shelf" MD programs, the components of the pressure tensor, $P_{i i}$ are calculated explicitly 
using the virial (mechanical) route, hence its use herein with no prejudice towards the test area method. Assuming a two-phase system with a clear interface, the tension is proportional to the difference between the normal ( $z$ direction) and the tangential components ( $x-y$ direction) of the pressure tensor:

$$
\gamma=\frac{1}{n} \int_{0}^{L z}\left[P_{z z}-\frac{P_{x x}+P_{y y}}{2}\right] d z
$$

where $L_{z}$ is the longest length of the simulation cell and $n$ is the number of surfaces. ${ }^{38}$

From the molecular dynamics perspective a direct calculation of the equilibrium surface tension as a function of the surface excess can be performed independently of the surfactant concentration. Since the system is very dilute (bulk concentrations are typically in the order of $10^{-1} \mathrm{~mol} \mathrm{~m}^{-3}$ ) in a small simulation cell, the number of free surfactants in solution away from the interface is negligible. Hence one can focus on the number of molecules on the surface, which in essence will be numerically equivalent to $\Gamma$, from where the bottom-right-hand plot in figure 1 can be drawn.

\section{Results and discussions}

Figure 3 shows the calculated surface tension for both the cis and trans versions of the surfactant as compared with experimental data. ${ }^{42}$ As expected, the surface tension decreases monotonically as more surfactant molecules are added to the interface. For the cis isomer, a critical point is attained when the concentration of surfactants at the surface reaches $\Gamma_{\max }=4.61 \times 10^{-6} \mathrm{~mol} \mathrm{~m}^{-2}$ (corresponding to 100 surfactant molecules per surface in the periodic cell) associated to a $\gamma_{\min }=41.6 \mathrm{mN} \mathrm{m}^{-1}$. The maximum surface excess and the maximum lowering of surface tension are in excellent agreement with the experimental ${ }^{42}$ 
values at $4.6 \times 10^{-6} \mathrm{~mol} \mathrm{m^{-2 }}$ and $40.6 \mathrm{mN} \mathrm{m}^{-1}$ respectively. For the trans isomer, the prediction is of similar quality with $\Gamma_{\max }=6.46 \times 10^{-6} \mathrm{~mol} \mathrm{~m}{ }^{-2}$ which compares well with the experimental value ${ }^{42}$ of $7.6 \times 10^{-6} \mathrm{~mol} \mathrm{~m}^{-2}$ corresponding to 140 surfactant molecules per simulation cell surface associated to a $\gamma_{\min }=37.2 \mathrm{mN} \mathrm{m}^{-1}$ (experimental value is $29.5 \mathrm{mN}$ $\left.\mathrm{m}^{-1}\right)^{42}$. It is worth reaffirming that the simulations as reported are incapable of determining the actual concentration of the cmc. It is here that a link to experiments must be made, i.e. by mapping the surface excess in both the model and the experiment.

If surfactant concentrations above the surface excess are considered, the systems will surpass the saturation of the interface, and the excess surfactant molecules will not reside directly at the interface but rather above it (as a bilayer) or will cooperatively "sink" in the aqueous phase forming aggregates. However, the computation of the surface tension on these simulation cells, either by the virial (mechanical) route or by the thermodynamic route will provide a numerical answer. This result is spurious and cannot be considered a true surface tension, as the assumption behind the methodology is that the interfacial area is confined to (in this case) a fixed square area in the $x-y$ plane. The final saturation point of the system cannot be deduced without either visually inspecting the configurations to rule out the formation of micelles and or a new phase, or by monitoring the positions of the surfactant molecules in the simulation box. 


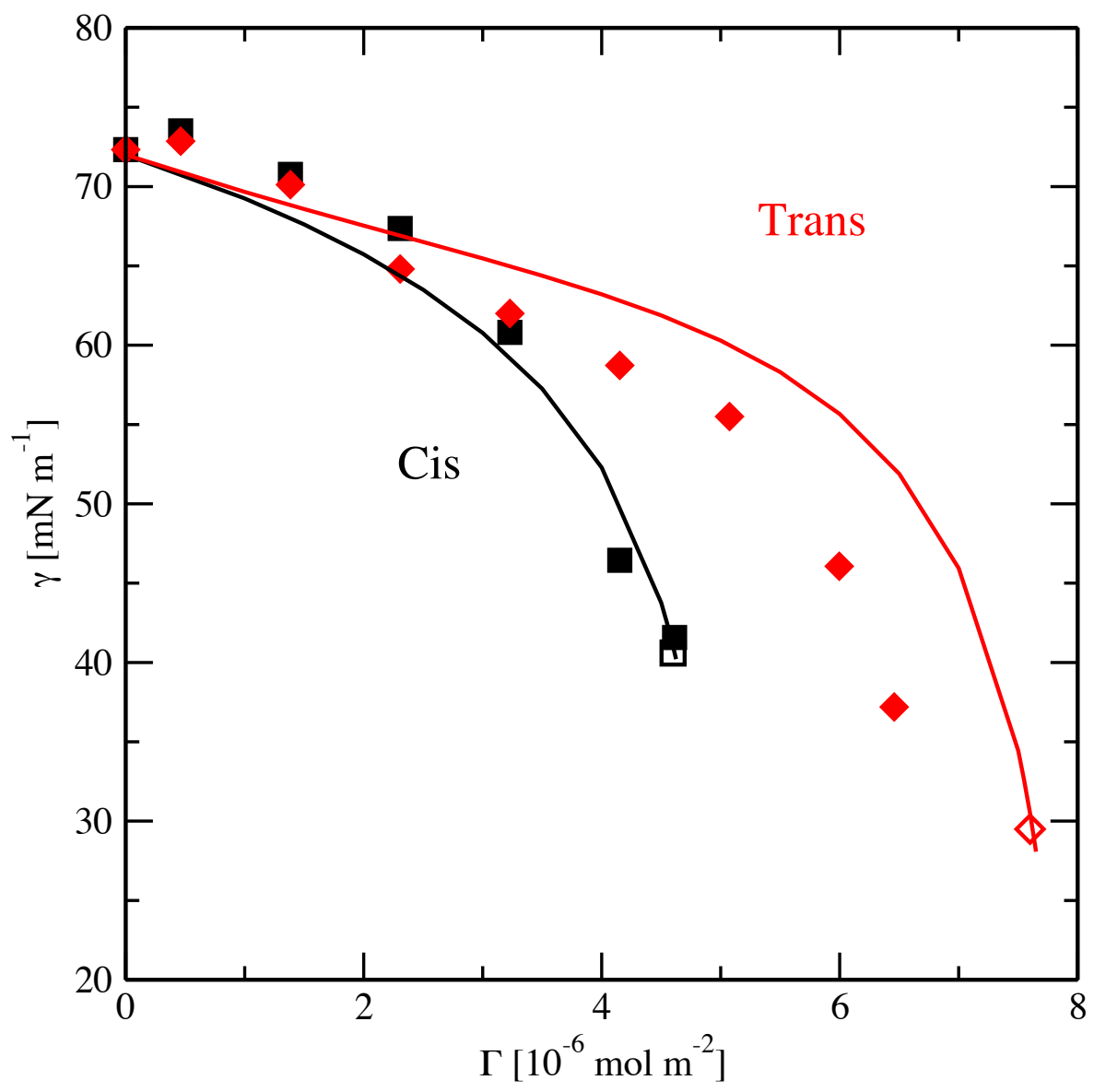

Figure 3. Surface tension as a function of surface excess for cis (black) and trans (red) isomers. Solid lines are smoothed experimental ${ }^{42}$ data. Experimental saturated surface limits are highlighted as an open square and diamond for cis and trans, respectively. Solid squares and diamonds are simulations for cis and trans isomers, respectively.

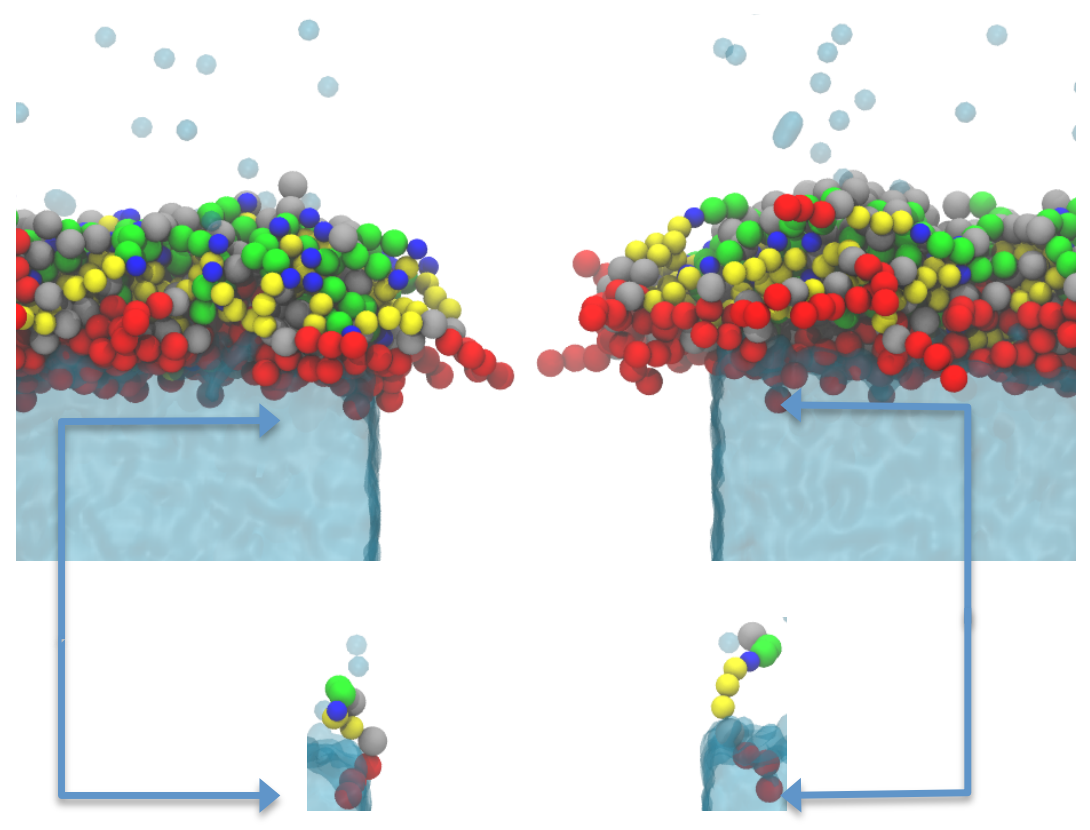


Figure 4. Snapshots of representative equilibrium configurations at $\Gamma=4.15 \times 10^{-6} \mathrm{~mol} \mathrm{~m}^{-2}$ for the cis (left) and trans (right) isomers. Only a section of the simulation cell is shown; water molecules in the bulk are blurred for clarity. Color-coding of the surfactant follows the theme of figure 2. The bottom cartoon shows the conformation of single cis and trans surfactant molecule extracted from the surface.

One must recognize that the high quality in the agreement between the tensions of the model cis surfactant and the experiments is somewhat determined by the adjustment of the cross energy parameters involving water. Within the SAFT force field, the parameterisation of water is the one with most uncertainties, as considering a non-polar isotropic bead is a very crude approximation for the intricacies of molecular water interactions. However, no further fitting is performed for the trans version, i.e. the same parameter set is used. The change in the tension is due exclusively to the conformations brought about by the extension of the internal angle. The model captures the change in a natural way and exemplifies the entropic nature of the transformation.

The nature of the CG simulations allow the monitoring of molecular conformations at the interface. Figure 4 shows the cis and trans isomers at a surface concentration of $4.15 \times 10^{-6} \mathrm{~mol} \mathrm{~m}^{-2}$ (with 90 surfactant molecules per simulation cell surface). One can notice the clear effect of the azo-benzene switch on the final conformation of the isomers at the water surface. The effect of the trans conformation of the azo-benzene is twofold: a) it "stretches" the molecule in such a way that the hydrophilic moieties between the azo group and the terminal hydrophobic alkane become, on average, less accessible to the water, and b) it allows for a more compact packing of the surfactants at the interface. A consequence of the above is that the molecular layer occupied by the trans conformers is thicker and more populated than the cis ones, reflected in an increase in the $\mathrm{cmc}$ and in the maximum surface excess. 
An angle analysis is performed to determine the effect of the azo-switch over the general orientation of the surfactant population with respect to the water surface. Accordingly, two molecular vectors are defined. The first one, $\mathbf{C}$, monitors the hydrophilic section of the surfactant, and is oriented along the length of the carbitol group, between the initial and final carbon bead. The second vector, $\mathbf{A}$, is defined along the anisol moiety and describes an important section of the hydrophobic part of the molecule. The azo group is buried within the hydrophobic half of the surfactant and changes the conformation of this sector. The average orientation of these vectors with respect to the surface, in terms of the smallest angle $\alpha$ between each vector and the plane of the water surface, is tracked during an equilibrated trajectory for $10 \mathrm{~ns}$. The results of the angle analysis for $\Gamma=4.15 \times 10^{-6} \mathrm{~mol}$ $\mathrm{m}^{-2}$ can be seen in figure 5 . 

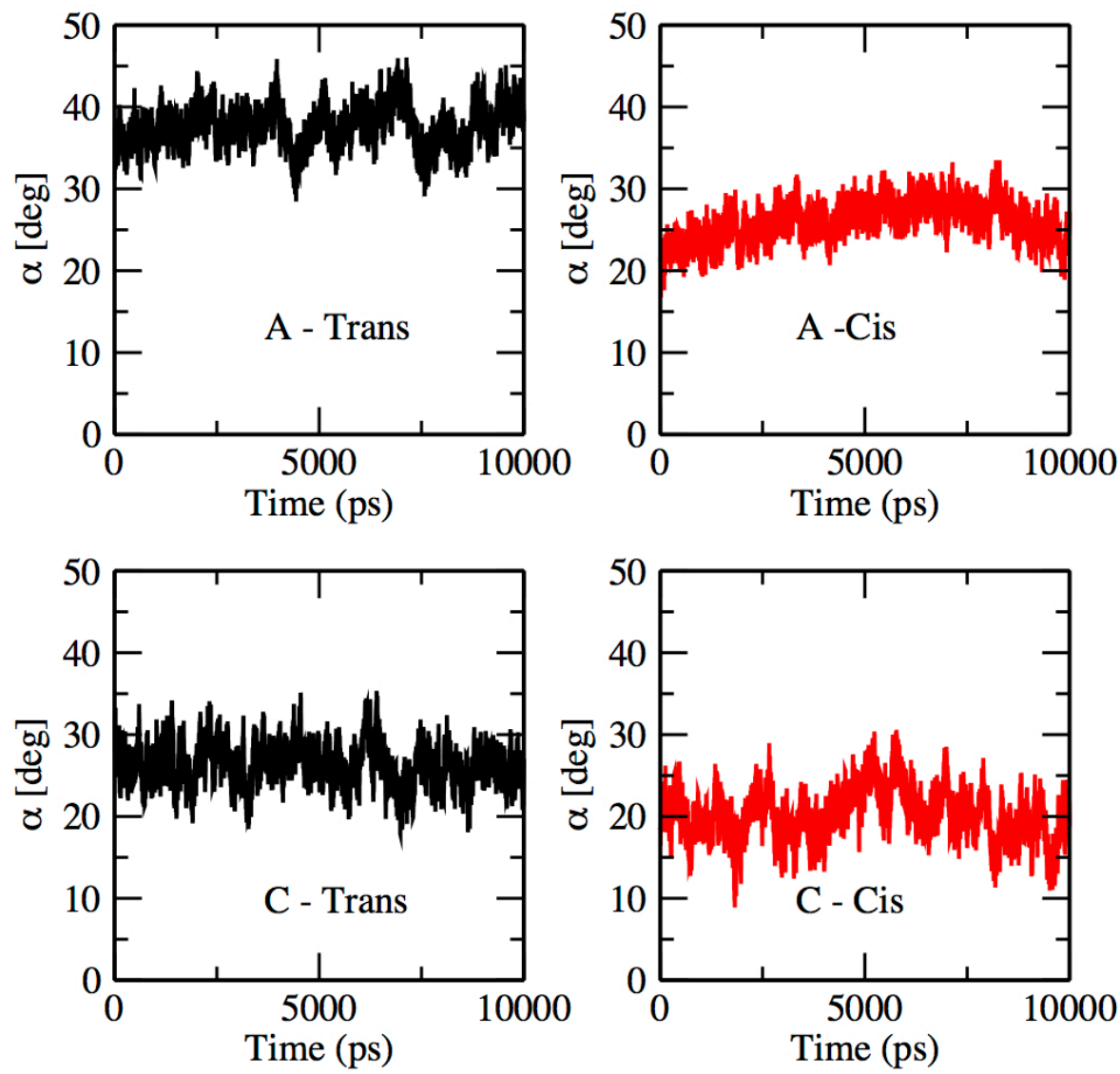

Figure 5. Angle between the water surface plane and the anisole vector A (top) and the carbitol vector C (bottom), for the trans (left plots) and cis (right plots) surfactants.

For the trans conformations, the angles are larger than for the cis conformations, indicating that, on average, the trans molecules are in a more upright orientation (normal to the water interface). The anisol vectors, as representative components of the hydrophobic part of the surfactant, went from an average value of $37.64^{\circ}$ (with a standard deviation of $\left.2.57^{\circ}\right)$ to $26.02^{\circ}\left[2.39^{\circ}\right]$ when considering the transition from trans to cis. Meanwhile, under the same conditions the carbitol vectors (hydrophilic) changed in average from $26.31^{\circ}\left[2.63^{\circ}\right]$ to $20.24^{\circ}[3.13]$ when comparing the trans vs. the cis conformations. 


\section{Conclusions}

An alternative simulation method to explore the pre-cmc region of a surfactant at the air-water interface, based on the calculation of the surface tension in terms of the surface excess values, is presented. Although the underlying simulations, where a given number of surfactants are placed in a periodic box with a section of a planar water-vapour interface, are not uncommon, as far as we are aware there has been no attempt to systematically relate the simulations to the adsorption isotherms commonly produced from experiments. In the simulations, surface tensions may be calculated directly, however, any attempt to directly relate them to actual bulk fluid concentrations is futile. The key assumption here is that the bulk liquid phase is extremely dilute, to the point that for the relatively small sample studied, in a statistical sense, there will be no surfactant molecules immersed in the fluid. It follows from this that all the surfactants in the simulation cell will form part of the interfacial region and the computation of the surface excess is then straightforward. This surface excess can be extracted directly from the experiments and is the quantity to be used to link simulations to experiments.

We have exemplified the concept by exploring the surface activity of a coarse grained model of a light switching surfactant. The use of physically-based coarse grained approaches, such as the SAFT- $\gamma$ forcefield, not only allow for the exploration of meaningful system sizes and times, but also provides quantitative predictions in terms of the efficiency, effectiveness and functionality of aqueous surfactant solutions.

The molecular picture provided by the simulations dissipates some of the speculations with respect to the conformations adopted by the cis and trans versions of the particular surfactant chosen in this study. There is clear evidence that the break in symmetry induced by the azo group is the sole driver for the difference in the surface 
tension seen between the two versions of the amphiphile. The cis group creates a "kink" in the hydrophobic section of the molecule, which hinders the optimal packing of the surfactant at the interface. The inability of the cis conformation to reach a high packing at the interface impedes the attainment of very low surface tensions and high surface excesses. These observations are commensurate with the observed macroscopic evidence and shed light on the mechanism of light-switching surfactancy.

Both the simulation methods described herein and the coarse grained methodologies can be employed with other types of ionic and non-ionic surfactants and can become a tool for surfactant screening.

\section{Acknowledgments}

This work was supported by the Engineering and Physical Sciences Research Council EPSRC (U.K.) through research grants (EP/I018212, EP/J014958 and EP/J010502). Experimental data was kindly supplied by Prof. T.A. Hatton. Simulations described herein were performed using the facilities of the Imperial College High Performance Computing Service. 


\section{References}

1 J.N. Israelachvili, Intermolecular and surface forces, $2^{\text {nd }}$ ed. Harcourt Brace, London, 1992 (ISBN 10: 0123751810 / 0-12-375181-0)

2 D.R. Karsa, Industrial applications of surfactants IV, Cambridge: RSC, 1999 (ISBN 10: 0854047735 / 0-85404-773-5)

3 J. Eastoe, Surfactant Aggregation and Adsorption at Interfaces, in Colloid Science: Principles, Methods and Applications (ed T. Cosgrove), Blackwell Publishing Ltd., Oxford, UK, 2009 (DOI: 10.1002/9781444305395.ch4)

4 M.J. Rosen, J.T. Kunjappu, Surfactants and interfacial phenomena, $4^{\text {th }}$ ed. Wiley, 2012 (ISBN: 978-0-470-54194-4)

5 D. Vollhardt, V.B. Fainerman, Characterisation of phase transition in adsorbed monolayers at the air/water interface, Advances in Colloid and Interface Science 2010, 154, 1-19. (DOI: 10.1016/j.cis.2010.01.003)

6 J. Eastoe, J.S Dalton, Dynamic surface tension and adsorption mechanisms of surfactants at the air-water interface, Advances in Colloid and Interface Science 2000, 85, 103-144. (DOI: 10.1016/S0001-8686(99)00017-2)

7 E.J. Maginn, J.R. Elliott, Historical Perspective and Current Outlook for Molecular Dynamics as a Chemical Engineering Tool, Industrial and Engineering Chemistry Research 2010, 49, 3059-3078. (DOI: 10.1021/ie901898k)

8 B. Creton, C. Nieto-Draghi, N. Pannacci, Prediction of Surfactants' Properties using Multiscale Molecular Modeling Tools: A Review Oil \& Gas Science and Technology - Rev. IFP Energies nouvelles 2012, 67(6), 969-982. (DOI: 10.2516/ogst/2012040)

9 E.S. Boek, A. Jusufi, H. Lowen, G.C. Maitland, Molecular design of responsive fluids: molecular dynamics studies of viscoelastic surfactant solutions, J. Phys. Condens. Matter 2002, 14, 9413-9430. (DOI: 10.1088/0953-8984/14/40/326) B. Jonsson, O. Edholm, O. Teleman, Molecular-dynamics simulations of a sodium octanoate micelle in aqueous-solution, J. Chem. Phys. 1986, 85, 2259-2271. (DOI: $10.1063 / 1.451122)$

11 K. Watanabe, M. Ferrario, M.L. Klein, Molecular-dynamics study of a sodium octanoate micelle in aqueous-solution, J. Phys. Chem. 1988, 92, 819-821. (DOI: $10.1021 / \mathrm{j} 100314 \mathrm{a} 045)$

12 K.J. Schweighofer, U. Essmann, M. Berkowitz, Simulation of sodium dodecyl sulfate at the water-vapor and water-carbon tetrachloride interfaces at low surface coverage, J. Phys. Chem. B 1997, 101, 3793-3799. (DOI: 10.1021/jp963460g)

J.B. Maillet, V. Lachet, P.V. Coveney, Large scale molecular dynamics simulation of self-assembly processes in short and long chain cationic surfactants, Phys. Chem. Chem. Phys. 1999, 1, 5277-5290.( DOI: 10.1039/A905216J) 
D.P. Tieleman, D. van der Spoel, H.J.C. Berendsen, Molecular dynamics simulations of dodecylphosphocholine micelles at three different aggregate sizes: Micellar structure and chain relaxation, J. Phys. Chem. B 2000, 104, 6380-6388. (DOI: 10.1021/jp001268f)

S.J. Marrink, D.P. Tieleman, A.E. Mark, Molecular dynamics simulation of the kinetics of spontaneous micelle formation, J. Phys. Chem. B 2000, 104, $12165-$ 12173. (DOI: 10.1021/jp001898h)

J. Shelley, K. Watanabe, M.L. Klein, Simulation of a sodium dodecyl-sulfate micelle in aqueous-solution, Int. J. Quantum Chem. 1990, 38, 103-117. (DOI: 10.1002/qua.560381713)

A.D. MacKerell, Molecular-dynamics simulation analysis of a sodium dodecylsulfate micelle in aqueous-solution - decreased fluidity of the micelle hydrocarbon interior, J. Phys. Chem. 1995, 99, 1846-1855. (DOI: 10.1021/j100007a011)

T. Bast, R. Hentschke, Molecular Dynamics Simulation of a Micellar System:

2,3,6,7,10,11-Hexakis(1,4,7-trioxaoctyl)triphenylene in Water, J. Phys. Chem.

1996, 100 (30), 12162-12171. (DOI: 10.1021/jp9537901)

C.D. Bruce, M.L. Berkowitz, L. Perera, M.D.E. Forbes, Molecular dynamics simulation of sodium dodecyl sulfate micelle in water: Micellar structural characteristics and counterion distribution, J. Phys. Chem. B 2002, 106, 3788-3793. (DOI: 10.1021/jp013616z)

A.R. Rakitin, G.R. Pack, Molecular dynamics simulations of ionic interactions with dodecyl sulfate micelles, J. Phys. Chem. B 2004, 108, 2712-2716. (DOI: 10.1021/jp030914i)

N. Yoshii, S. Okazaki, A molecular dynamics study of surface structure of spherical SDS micelles, Chem. Phys. Lett. 2006, 426, 66-70. (DOI:

10.1016/j.cplett.2006.05.038)

M. Sammalkorpi, M. Karttunen, M. Haataja, Structural properties of ionic detergent aggregates: a large-scale molecular dynamics study of sodium dodecyl sulphate, $J$. Phys. Chem. B 2007, 111, 11722-11733. (DOI: 10.1021/jp072587a)

D.W. Cheong, A.Z. Panagiotopoulos, Monte Carlo simulations of micellization in model ionic surfactants: Application to sodium dodecyl sulphate, Langmuir 2006, 22, 4076-4083. (DOI: 10.1021/la053511d)

W.M. Gelbart, A. BenShaul, The "new" science of "complex fluids", J. Phys. Chem. 1996, 100, 13169-13189. (DOI: 10.1021/jp9606570)

R. Goetz, R. Lipowsky, Computer simulations of bilayer membranes: Self-assembly and interfacial tension, J. Chem. Phys. 1998, 108, 7397-7409. (DOI:

$10.1063 / 1.476160)$ 
S. Bandyopadhyay, M.L. Klein, G.J. Martyna, M. Tarek, Molecular dynamics studies of the hexagonal mesophase of sodium dodecylsulphate in aqueous solution, Mol. Phys. 1998, 95, 377-384. (DOI: 10.1080/00268979809483170)

J.C. Shelley, M.Y. Shelley, Computer simulation of surfactant solutions, Curr. Opin. Colloid Interface Sci. 2000, 5, 101-110. (DOI: 10.1016/S13590294(00)00042-X)

A.H. de Vries, A.E. Mark, S.J. Marrink, The binary mixing behavior of phospholipids in a bilayer: A molecular dynamics study, J. Phys. Chem. B 2004, 108, 2454-2463. (DOI: 10.1021/jp0366926)

J. Gao, W. Ge, G. Hu, J. Li, From homogeneous dispersion to micelles - A molecular dynamics simulation on the compromise of the hydrophilic and hydrophobic effects of sodium dodecyl sulfate in aqueous solution, Langmuir 2005, 21, 5223- 5229. (DOI: 10.1021/1a047121n)

W. Shinoda, R. DeVane, M.L. Klein, Multi-property fitting and parameterization of a coarse grained model for aqueous surfactants, Mol. Simul. 2007, 33(1-2), 27-36. (DOI:10.1080/08927020601054050)

G. Mohan, D.I. Kopelevich, A multiscale model for kinetics of formation and disintegration of spherical micelles, J. Chem. Phys. 2008, 128, 044905. (DOI: $10.1063 / 1.2823729)$

F. Sterpone, G. Briganti, C. Pierleoni, Sphere versus Cylinder: The Effect of Packing on the Structure of Nonionic C12E6 Micelles, Langmuir 2009, 25, 89608967. (DOI: 10.1021/la900591e)

P. Alexandridis, J.F. Holzwarth, T.A. Hatton, Interfacial Dynamics of Water-in-Oil Microemulsion Droplets - Determination of the Bending Modulus using Iodine Laser Temperature-Jump, Langmuir 1993, 9, 2045- 2052. (DOI: 10.1021/la00032a024)

A. Jusufi, S. Sanders, M.L. Klein, A.Z. Panagiotopoulos, Implicit-solvent models for micellization: Nonionic surfactants and temperature-dependent properties, $J$. Phys. Chem. B 2011, 115, 990-1001. (DOI: 10.1021/jp108107f)

S. Bandyopadhyay, M. Tarek, M.L. Lynch, M.L. Klein, Molecular dynamics study of the poly(oxyethylene) surfactant C12E2 and water, Langmuir 2000, 16, 942-946. (DOI: 10.1021/la9909493)

F. Sterpone, G. Briganti, C. Pierleoni, Molecular dynamics study of spherical aggregates of chain molecules at different degrees of hydrophilicity in water solution, Langmuir 2001, 17(16), 5103- 5110. (DOI: 10.1021/la000750m)

S. Garde, L. Yang, J.S. Dordick, M.E. Paulaitis, Molecular dynamics simulation of C8E5 micelle in explicit water: structure and hydrophobic solvation thermodynamics, Mol. Phys. 2002, 100(14), 2299-2306. (DOI: 10.1080/00268970110118312) 
W. Shinoda, R. DeVane, M.L. Klein, Coarse-grained molecular modeling of nonionic surfactant self-assembly, Soft Matter 2008, 4(12), 2454- 2462. (DOI: 10.1039/B808701F)

G. Barnes, I. Gentle, Interfacial Science: an Introduction, $2^{\text {nd }}$ edition Oxford, 2011 (ISBN-10: 019957118X)

S. Shinkai, K. Matsuo, A. Haradaand, O.Manabe, Photocontrol of micellar catalyses, J. Chem. Soc., Perkin Trans. 2 1982, 1261- 1265. (DOI: 10.1039/P29820001261)

J. Eastoe, A. Vesperinas, Self-assembly of light-sensitive surfactants. Soft Matter 2005, 1(5), 338- 347. (DOI: 10.1039/B510877M)

T. Shang, K.A. Smith, T.A. Hatton, Photoresponsive surfactants exhibiting unusually large, reversible surface tension changes under varying illumination conditions, Langmuir 2003, 19, 10764-10773. (DOI: 10.1021/la0350958)

E.A. Muller, G. Jackson, Force field parameters from the SAFT- $\gamma$ equation of state for use in coarse-grained molecular simulation, Ann. Rev. Chem . Biomol. Eng. 2014, 5, 405-427. (DOI: 10.1146/annurev-chembioeng-061312-103314)

T. Lafitte, C. Avendaño, V. Papaioannou, A. Galindo, C.S. Adjiman, G. Jackson, E.A. Müller, SAFT-gamma force field for the simulation of molecular fluids: 3 . Coarse-grained models of benzene and hetero-group models of n-decylbenzene, Molecular Physics 2012, 110 (11-12), 1189-1203. (DOI:10.1080/00268976.2012.662303)

C. Avendaño, T. Lafitte, A. Galindo, C.S. Adjiman, G. Jackson, E.A. Müller, SAFT-gamma force field for the simulation of molecular fluids. 1. A single-site coarse grained model of carbon dioxide, J. Phys. Chem. B 2011, 115(38), 1115411169. (DOI: DOI: 10.1021/jp204908d)

C. Avendaño, T. Lafitte, C.S. Adjiman, A. Galindo, E.A. Müller, G. Jackson, SAFT- $\gamma$ Force Field for the Simulation of Molecular Fluids: 2. Coarse-Grained Models of Greenhouse Gases, Refrigerants, and Long Alkanes, J Phys Chem B. 2013, 117(9), 2717-2733. (DOI: 10.1021/jp306442b) O. Lobanova, PhD. Thesis. Imperial College London, 2014

A. Mejia, C. Herdes, E.A. Müller, Force fields for coarse-grained molecular simulations from a corresponding states correlation. Ind. Eng. Chem. Res.2014, 53, 4131-4141. (DOI: 10.1021/ie404247e) GROMACS: fast, flexible, and free, J. Comput. Chem. 2005, 26(16), 1701-1718. (DOI: $10.1002 /$ jcc.20291)

S.J. Marrink, H.J. Risselada, S. Yefimov, D.P. Tieleman and A.H. de Vreis, The MARTINI Force Field: Coarse Grained Model for Biomolecular Simulations, $J$. Phys. Chem. 2007, 111, 7812-7824. (DOI: 10.1021/jp071097f) 
G. J. Gloor, G. Jackson, F. J. Blas, E. de Miguel, Test-area simulation method for the direct determination of the interfacial tension of systems with continuous or discontinuous potentials, J. Chem. Phys. 2005, 123, 134703. (DOI:

10.1063/1.2038827)

52 J.G. Sampayo, A. Malijevský, E.A. Müller, E. de Miguel, G. Jackson, Communications: Evidence for the role of fluctuations in the thermodynamics of nanoscale drops and the implications in computations of the surface tension, $J$ Chem Phys. 2010, 132, 141101. (DOI: 10.1063/1.3376612) 